\title{
The novel noise classification techniques found on quadruple threshold statistical detection filter under fix intensity impulse outlier environment
}

\author{
Vorapoj Patanavijit, Kornkamol Thakulsukanant
}

Faculty of Engineering, Assumption University of Thailand, Thailand

\section{Article Info}

Article history:

Received Dec 7, 2020

Revised May 21, 2021

Accepted Aug 30, 2021

\section{Keywords:}

Digital image denoising Digital image processing Quadruple threshold statistical detection

Standard median filter Triple threshold statistical detection

ABSTRACT

Because of the enormous necessity of contemporary noise suppressing algorithms, this article proposes the novel noise classification technique found on QTSD filter improved from the TTSD filter. The four thresholds for each auxiliary situations are incorporated into the proposed QTSD framework for dealing with the limitation of the earlier noise classification technique. The mathematical pattern is modeled by each photograph elements and is investigated in contradiction to the $1^{\text {st }}$ threshold for analyzing whether it is non-noise or noise photograph elements. Subsequently, the calculated photograph element is analyzed with the contradiction between the $2^{\text {nd }}$ threshold, which is modeled by using the normal distribution (mean and variance), and is analyzed with the contradiction between the $3^{\text {rd }}$ threshold, which is modeled by using the quartile distribution (median). Finally, the calculated photograph element is investigated in contradiction to the 4th threshold, which is modeled from maximum or minimum value for analyzing whether it is non-noise or noise photograph elements FIIN. For performance evaluation, extensive noisy photographs are made up of nine photographs under FIIN environment distribution, which are synthesized for investigating the proposed noise classification techniques found on QTSD filter in the objective indicators (noise classification, non-noise classification and overall classification correctness). From these results, the proposed noise classification technique can outstandingly produce the higher correctness than the earlier noise classification techniques.
\end{abstract}

This is an open access article under the $\underline{C C B Y-S A}$ license.

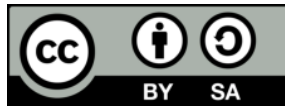

\section{Corresponding Author:}

Vorapoj Patanavijit

Faculty of Engineering

Assumption University of Thailand

VME Bldg., 2nd Flr., 88 Moo 8 Bang Na-Trad Km. 26, Bangsaothong, Samuthprakarn 10540, Thailand

Email: patanavijit@yahoo.com

\section{INTRODUCTION}

In the course of twenty-five years, almost contemporary advance noise suppressing algorithms [1]-[5], which are commonly incorporated of two fundamental procedures: the noise classification technique and the noise recovery technique, have been extensively investigated and developed from the extensively implemented domains [6]-[9]. As a result, the noise classification technique is highly dominant to the overall effectiveness of noise suppressing algorithms thereby extensive advance noise suppressing algorithms [10]-[22] are investigated and developed for both noise classification techniques and noise recovery techniques as following. In 1975, the noise suppressing algorithm found on a standard median filter (SMF) [20] was initially introduced for (fix intensity impulse outlier) FIIN thus the SMF is one of the practical 
complexity with acceptable effectiveness. For recovering FIIN on color electronic photographs in 1990, the vector median filter (VMF) [10], which is improved from SMF, is proposed and, late, the VMF will become of one the high effective noise suppressing algorithm for color electronic photographs. The noise suppressing algorithm [11] based on intensity preserving cast removal and particle swarm optimization for color images was proposed in 2017. Consequently, automatic computer-assisted diagnosis algorithm [12] based on SVM classification for MRI brain images was proposed in 2017. In 2018, the noise suppressing algorithm [13] based on noise level estimation using local statistics was proposed for digital Image. Next, the noise suppressing algorithm [14] based on an efficient filtering technique was proposed for color images in 2018. Later, the classical noise suppressing algorithm based on Wiener and Gaussian filtering [15] been analyzed its performance by varying kernel size in 2019. Subsequently, the noise suppressing algorithm based on filter technique [16] been analyzed its performance on medical images in 2019. Bilal Charmouti et al. [17] reviews many noise suppressing algorithms based on different fundamental techniques and comparatively simulates these performances on denoising perspective in 2019. The modified noise suppressing algorithms based on a fractional wavelet technique [18], which is the extended Haar wavelet as fractional order by a low-pass filtering generalization with the fractional delay process, has been proposed in 2020. In 2020, the noise suppressing algorithm based on discrete wavelet transform [19] using with noise density estimation was proposed for underwater acoustic noise. Next, the noise suppressing algorithm found on an adaptive median filter (AMF) [21], which was developed from the SMF for automatically adjusting the computational area, was introduced for FIIN in 1994 therefore the AMF is one of the practical complexity with high effectiveness. Many modern noise suppressing algorithms [22]-[25], which are traditionally comprised of the noise classification technique and the noise recovery technique, have been proposed as following. A hybrid statistical noise suppression technique [22] has been proposed for suppressing impulse noise in 2017. Next, another effective noise suppressing algorithm, adaptive decision based inverse distance weighted interpolation (DBIDWI) algorithm [23], [24], which was proposed for high density salt and pepper noise by V. Kishorebabu et al. [24] in 2017, has been analyzed its performance [23] for suppressing impulse noise for all density salt and pepper noise in 2019. The modern noise classification techniques [25] such as ROAD, ROLD and RORD are comparatively analyzed for detecting impulse noise in 2019. Later, the noise suppressing algorithm found on the triple threshold statistical detection (TTSD) filter [26] in 2018 was introduced and the noise suppressing algorithm has very impressive effectiveness for random intensity impulse noise (RIIN). As a results, the TTSD has been analyzed its performance [27] for suppressing impulse noise for all density salt and pepper noise in 2019. Improved from the outstanding TTSD filter, this article proposes the novel noise classification technique found on QTSD for FIIN [28].

\section{THE PROPOSED QTSD FILTER FOR THE NOISE CLASSIFICATION TECHNIQUE}

Delineate that $\boldsymbol{x}$ is the non-noise photograph, which are consolidated of an photograph element $x_{i, j}$ at point $(i, j)$, where $0 \leq x_{i, j} \leq 255$ or $[0,255]$ is characterized as the magnitude space of this photograph. Next, $\boldsymbol{y}$ is an noise photograph that are consolidated of an noise photograph element $y_{i, j}$. As a results, $y_{i, j}$ can be conceptually declared as statement.

$$
y_{i, j}=\left\{\begin{array}{cc}
0 & \text { atprobability } p \\
255 & \text { atprobability } q \\
x_{i, j} & \text { atprobability }(1-p-q)
\end{array}\right.
$$

where $(p-q)$ is the FIIN denseness

The conceptual process of the QTSD filter [28] for classifying the impulsive noise can be declared as subprocess:

a. Declare the filter size to be $5 \times 5(A)$ at center $y_{i, j}$ and, later, estimate the first statistical moment $\left(\mu_{A}\right)$ and the second statistical moment $\left(\sigma_{A}\right)$ of each elements within window area $A$ except the center element.

b. Estimate the absolute subtraction $\left(p_{i j}\right)$ of each elements in this $A$ window and $\mu_{A}$, later, write down 24 enumerated values $\left(p_{i j}\right)$.

c. Estimate the first statistical moment $\left(\mu_{P}\right)$ and the second statistical moment $\left(\sigma_{P}\right)$ of each 24 enumerated values $\left(p_{i j}\right)$.

$$
T_{1}=\mu_{P}+\sigma_{P}
$$


d. Estimate the absolute subtraction $q_{i j}$ of the center element $y_{i, j}$ and other 24 neighbor elements in this $A$ window.

e. Estimate the first statistical moment $\left(\mu_{q}\right)$ from all absolute subtraction $q_{i j}$ and identify the noise signature $(N S)$ as

$$
N S=\mu_{q}
$$

f. The $y_{i, j}$ will be detected as outlier element where $N S \geq T_{1}$ and $\left(\left(y(i, j)=T_{4 \min }()\right)\right.$ or $(y(i, j)=$ $\left.T_{4 \max }(\mathrm{)})\right)$ otherwise go to sub-process 7 .

g. Estimate the second constant $T_{2}$ that is characterized by the Gaussian distribution model (the first and the second statistical moment) as

$$
\begin{array}{r}
T A_{A_{2 \text { min }}} \\
\text { and } \\
T A_{A_{2 \text { max }}}
\end{array}
$$

h. The $y_{i, j}$ is characterized as a outlier element where If $\left(y_{i, j} \leq T_{2 \min }\right.$ or $\left.y_{i, j} \geq T_{2 \max }\right)$ and $((y(i, j)=$ $\left.T_{4 \min }()\right)$ or $\left.\left(y(i, j)=T_{4 \max }()\right)\right)$ otherwise go to sub-process 9 .

i. $\quad$ Estimate the third constant $T_{3}$ that is characterized by quartile distribution model (median) as

$$
T 1_{3 \min }
$$

and

$$
T 3_{3 \max }
$$

j. $\quad$ The $y_{i, j}$ is characterized as a outlier element where $\left(y_{i, j} \leq Q_{1}\right.$ or $\left.y_{i, j} \geq Q_{3}\right)$ and $\left(\left(y(i, j)=T_{4 \min }()\right)\right.$ or $\left.\left(y(i, j)=T_{4 \max }()\right)\right)$ otherwise $y_{i, j}$ is a characterized as genuine element.

In order to illustrate the noise classification process of the QTSD filter, the extensive sub-process of the proposed noise classification technique can be illustrated as Figure 1 in appendix.

\section{EXPERIMENTAL SIMULATION RESULTS}

The experimental simulation investiages the QTSD filter in noise classification performance (in the first section) and overall noise suppressing performance (in the second section). The MATLAB software is utilized in these simulation, which are implemented on workstations with the hardware condition: Intel i76700HQ CPU with 16 GB main memory where each workstation is executed on different extensive noisy photographs under FIIN distributions. In these first experimental simulation, the correctness performance of the proposed QTSD filter are analyszed on nine noise photographs (Lena, M-calendar, Pepper, Pentagon, Girl, Mandrill, House, Airplane), which are caused by FIIN with bountiful noise denseness from 5\% to $90 \%$. The classification correctness of noise elements shall be conceptually declared as following statement.

$$
\operatorname{Acc}_{\text {noisy }}=\sum_{\substack{\forall \text { noisy } \\ \text { pixels }}}\left(\hat{y}_{\text {estimated noisy pixels }} / y_{\text {noisy pixels }}\right)
$$

The classification correctness of non-noise elements shall be conceptually declared as following statement.

$$
\mathrm{Acc}_{\text {noiseless }}=\sum_{\substack{\forall \text { noiseless } \\ \text { pixels }}}\left(\hat{y}_{\text {estimated noiseless pixels }} / y_{\text {noiseless pixels }}\right)
$$

The overall classification correctness of both noise and non-noise elements shall be experimentally characterized as upcoming actuarial calculation comment. 


$$
\operatorname{Accc}_{\text {overall }}=\left(\frac{1}{2} \sum_{\substack{\forall \text { noisy } \\ \text { pixels }}}\left(\frac{\hat{y}_{\text {estimated noisy pixels }}}{y_{\text {noisy pixels }}}\right)+\frac{1}{2} \sum_{\substack{\forall \text { noiseless } \\ \text { pixels }}}\left(\frac{\hat{y}_{\text {estimated noiseles pixels }}}{y_{\text {noiseless pixels }}}\right)\right)
$$

These simulation results of QTSD filtering classification correctness of noise elements, non-noise elements and overall noise and non-noise elements for noise denseness, which is correlatively analyzed with the recent powerful TTSD filter (that is proposed in 2018), from 5\% to $90 \%$ are illustrated in Table 1, Table 2 and Table 3 , respectively.

Table 1. The simulated result of noise classification correction of the proposed QTSD filter

\begin{tabular}{|c|c|c|c|c|c|c|c|c|c|}
\hline \multirow{2}{*}{ Tested Images } & \multicolumn{9}{|c|}{ FIIN Noise Denseness (\%) } \\
\hline & 5 & 10 & 15 & 20 & 25 & 30 & 35 & 40 & 45 \\
\hline Lena (TTSD) & 1.0000 & 1.0000 & 1.0000 & 1.0000 & 1.0000 & 1.0000 & 1.0000 & 1.0000 & 1.0000 \\
\hline Lena (QTSD) & 1.0000 & 1.0000 & 1.0000 & 1.0000 & 1.0000 & 1.0000 & 1.0000 & 1.0000 & 1.0000 \\
\hline M-cal (TTSD) & 1.0000 & 1.0000 & 1.0000 & 1.0000 & 1.0000 & 1.0000 & 1.0000 & 1.0000 & 1.0000 \\
\hline M-cal (QTSD) & 1.0000 & 1.0000 & 1.0000 & 1.0000 & 1.0000 & 1.0000 & 1.0000 & 1.0000 & 1.0000 \\
\hline Pepper (TTSD) & 1.0000 & 1.0000 & 1.0000 & 1.0000 & 1.0000 & 1.0000 & 1.0000 & 1.0000 & 1.0000 \\
\hline Pepper (QTSD) & 1.0000 & 1.0000 & 1.0000 & 1.0000 & 1.0000 & 1.0000 & 1.0000 & 1.0000 & 1.0000 \\
\hline Penta (TTSD) & 1.0000 & 1.0000 & 1.0000 & 1.0000 & 1.0000 & 1.0000 & 1.0000 & 1.0000 & 1.0000 \\
\hline Penta (QTSD) & 1.0000 & 1.0000 & 1.0000 & 1.0000 & 1.0000 & 1.0000 & 1.0000 & 1.0000 & 1.0000 \\
\hline Girl (TTSD) & 1.0000 & 1.0000 & 1.0000 & 1.0000 & 1.0000 & 1.0000 & 1.0000 & 1.0000 & 1.0000 \\
\hline Girl (QTSD) & 1.0000 & 1.0000 & 1.0000 & 1.0000 & 1.0000 & 1.0000 & 1.0000 & 1.0000 & 1.0000 \\
\hline Resolution (TTSD) & 1.0000 & 1.0000 & 1.0000 & 1.0000 & 1.0000 & 1.0000 & 1.0000 & 1.0000 & 1.0000 \\
\hline Resolution (QTSD) & 1.0000 & 1.0000 & 1.0000 & 1.0000 & 1.0000 & 1.0000 & 1.0000 & 1.0000 & 1.0000 \\
\hline Mandrill (TTSD) & 1.0000 & 1.0000 & 1.0000 & 1.0000 & 1.0000 & 1.0000 & 1.0000 & 1.0000 & 1.0000 \\
\hline Mandrill (QTSD) & 1.0000 & 1.0000 & 1.0000 & 1.0000 & 1.0000 & 1.0000 & 1.0000 & 1.0000 & 1.0000 \\
\hline House (TTSD) & 1.0000 & 1.0000 & 1.0000 & 1.0000 & 1.0000 & 1.0000 & 1.0000 & 1.0000 & 1.0000 \\
\hline House (QTSD) & 1.0000 & 1.0000 & 1.0000 & 1.0000 & 1.0000 & 1.0000 & 1.0000 & 1.0000 & 1.0000 \\
\hline Airplane (TTSD) & 1.0000 & 1.0000 & 1.0000 & 1.0000 & 1.0000 & 1.0000 & 1.0000 & 1.0000 & 1.0000 \\
\hline Airplane (QTSD) & 1.0000 & 1.0000 & 1.0000 & 1.0000 & 1.0000 & 1.0000 & 1.0000 & 1.0000 & 1.0000 \\
\hline Average (TTSD) & 1.0000 & 1.0000 & 1.0000 & 1.0000 & 1.0000 & 1.0000 & 1.0000 & 1.0000 & 1.0000 \\
\hline Average (QTSD) & 1.0000 & 1.0000 & 1.0000 & 1.0000 & 1.0000 & 1.0000 & 1.0000 & 1.0000 & 1.0000 \\
\hline \multirow{2}{*}{ Tested Images } & \multicolumn{9}{|c|}{ FIIN Noise Denseness (\%) } \\
\hline & 50 & 55 & 60 & 65 & 70 & 75 & 80 & 85 & 90 \\
\hline Lena (TTSD) & 1.0000 & 1.0000 & 1.0000 & 1.0000 & 1.0000 & 1.0000 & 1.0000 & 1.0000 & 1.0000 \\
\hline Lena (QTSD) & 1.0000 & 1.0000 & 1.0000 & 1.0000 & 1.0000 & 1.0000 & 1.0000 & 1.0000 & 1.0000 \\
\hline M-cal (TTSD) & 1.0000 & 1.0000 & 1.0000 & 1.0000 & 1.0000 & 1.0000 & 1.0000 & 1.0000 & 1.0000 \\
\hline M-cal (QTSD) & 1.0000 & 1.0000 & 1.0000 & 1.0000 & 1.0000 & 1.0000 & 1.0000 & 1.0000 & 1.0000 \\
\hline Pepper (TTSD) & 1.0000 & 1.0000 & 1.0000 & 1.0000 & 1.0000 & 1.0000 & 1.0000 & 1.0000 & 1.0000 \\
\hline Pepper (QTSD) & 1.0000 & 1.0000 & 1.0000 & 1.0000 & 1.0000 & 1.0000 & 1.0000 & 1.0000 & 1.0000 \\
\hline Penta (TTSD) & 1.0000 & 1.0000 & 1.0000 & 1.0000 & 1.0000 & 1.0000 & 1.0000 & 1.0000 & 1.0000 \\
\hline Penta (QTSD) & 1.0000 & 1.0000 & 1.0000 & 1.0000 & 1.0000 & 1.0000 & 1.0000 & 1.0000 & 1.0000 \\
\hline Girl (TTSD) & 1.0000 & 1.0000 & 1.0000 & 1.0000 & 1.0000 & 1.0000 & 1.0000 & 1.0000 & 1.0000 \\
\hline Girl (QTSD) & 1.0000 & 1.0000 & 1.0000 & 1.0000 & 1.0000 & 1.0000 & 1.0000 & 1.0000 & 1.0000 \\
\hline Resolution (TTSD) & 1.0000 & 1.0000 & 1.0000 & 1.0000 & 1.0000 & 1.0000 & 1.0000 & 1.0000 & 1.0000 \\
\hline Resolution (QTSD) & 1.0000 & 1.0000 & 1.0000 & 1.0000 & 1.0000 & 1.0000 & 1.0000 & 1.0000 & 1.0000 \\
\hline Mandrill (TTSD) & 1.0000 & 1.0000 & 1.0000 & 1.0000 & 1.0000 & 1.0000 & 1.0000 & 1.0000 & 1.0000 \\
\hline Mandrill (QTSD) & 1.0000 & 1.0000 & 1.0000 & 1.0000 & 1.0000 & 1.0000 & 1.0000 & 1.0000 & 1.0000 \\
\hline House (TTSD) & 1.0000 & 1.0000 & 1.0000 & 1.0000 & 1.0000 & 1.0000 & 1.0000 & 1.0000 & 1.0000 \\
\hline House (QTSD) & 1.0000 & 1.0000 & 1.0000 & 1.0000 & 1.0000 & 1.0000 & 1.0000 & 1.0000 & 1.0000 \\
\hline Airplane (TTSD) & 1.0000 & 1.0000 & 1.0000 & 1.0000 & 1.0000 & 1.0000 & 1.0000 & 1.0000 & 1.0000 \\
\hline Airplane (QTSD) & 1.0000 & 1.0000 & 1.0000 & 1.0000 & 1.0000 & 1.0000 & 1.0000 & 1.0000 & 1.0000 \\
\hline Average (TTSD) & 1.0000 & 1.0000 & 1.0000 & 1.0000 & 1.0000 & 1.0000 & 1.0000 & 1.0000 & 1.0000 \\
\hline Average (QTSD) & 1.0000 & 1.0000 & 1.0000 & 1.0000 & 1.0000 & 1.0000 & 1.0000 & 1.0000 & 1.0000 \\
\hline
\end{tabular}

Table 2. The simulated result of non-noise classification correction of the QTSD filter

\begin{tabular}{lccccccccc}
\hline \multirow{2}{*}{ Tested Images } & \multicolumn{10}{c}{ FIIN Noise Denseness $(\%)$} \\
\cline { 2 - 10 }$y$ & 5 & 10 & 15 & 20 & 25 & 30 & 35 & 40 & 45 \\
\hline Lena (TTSD) & 1.0000 & 1.0000 & 1.0000 & 1.0000 & 1.0000 & 1.0000 & 1.0000 & 1.0000 & 1.0000 \\
Lena (QTSD) & 1.0000 & 1.0000 & 1.0000 & 1.0000 & 1.0000 & 1.0000 & 1.0000 & 1.0000 & 1.0000 \\
M-cal(TTSD) & 1.0000 & 1.0000 & 1.0000 & 1.0000 & 1.0000 & 1.0000 & 1.0000 & 1.0000 & 1.0000 \\
M-cal(QTSD) & 1.0000 & 1.0000 & 1.0000 & 1.0000 & 1.0000 & 1.0000 & 1.0000 & 1.0000 & 1.0000 \\
Pepper (TTSD) & 1.0000 & 1.0000 & 1.0000 & 1.0000 & 1.0000 & 1.0000 & 1.0000 & 1.0000 & 1.0000 \\
Pepper (QTSD) & 1.0000 & 1.0000 & 1.0000 & 1.0000 & 1.0000 & 1.0000 & 1.0000 & 1.0000 & 1.0000 \\
Penta (TTSD) & 1.0000 & 1.0000 & 1.0000 & 1.0000 & 1.0000 & 1.0000 & 1.0000 & 1.0000 & 1.0000 \\
Penta (QTSD) & 1.0000 & 1.0000 & 1.0000 & 1.0000 & 1.0000 & 1.0000 & 1.0000 & 1.0000 & 1.0000 \\
Girl (TTSD) & 0.7032 & 0.7054 & 0.7056 & 0.7032 & 0.6991 & 0.6934 & 0.6841 & 0.6747 & 0.6639 \\
Girl (QTSD) & 0.7491 & 0.7466 & 0.7433 & 0.7397 & 0.7370 & 0.7335 & 0.7295 & 0.7252 & 0.7191 \\
\hline
\end{tabular}


Table 2. The simulated result of non-noise classification correction of the QTSD filter (continue)

\begin{tabular}{|c|c|c|c|c|c|c|c|c|c|}
\hline Resolution (TTSD) & 0.1946 & 0.1837 & 0.1729 & 0.1628 & 0.1527 & 0.1400 & 0.1356 & 0.1241 & 0.1111 \\
\hline Resolution (QTSD) & 0.1957 & 0.1846 & 0.1738 & 0.1639 & 0.1535 & 0.1409 & 0.1364 & 0.1246 & 0.1118 \\
\hline Mandrill (TTSD) & 0.9987 & 0.9988 & 0.9989 & 0.9991 & 0.9990 & 0.9991 & 0.9991 & 0.9992 & 0.9991 \\
\hline Mandrill (QTSD) & 0.9994 & 0.9994 & 0.9995 & 0.9996 & 0.9995 & 0.9995 & 0.9995 & 0.9996 & 0.9995 \\
\hline House (TTSD) & 1.0000 & 1.0000 & 1.0000 & 1.0000 & 1.0000 & 1.0000 & 1.0000 & 1.0000 & 1.0000 \\
\hline House (QTSD) & 1.0000 & 1.0000 & 1.0000 & 1.0000 & 1.0000 & 1.0000 & 1.0000 & 1.0000 & 1.0000 \\
\hline Airplane (TTSD) & 1.0000 & 1.0000 & 1.0000 & 1.0000 & 1.0000 & 1.0000 & 1.0000 & 1.0000 & 1.0000 \\
\hline Airplane (QTSD) & 1.0000 & 1.0000 & 1.0000 & 1.0000 & 1.0000 & 1.0000 & 1.0000 & 1.0000 & 1.0000 \\
\hline Average(TTSD) & 0.8774 & 0.8764 & 0.8753 & 0.8739 & 0.8723 & 0.8703 & 0.8688 & 0.8664 & 0.8638 \\
\hline Average(QTSD) & 0.8827 & 0.8812 & 0.8796 & 0.8781 & 0.8767 & 0.8749 & 0.8739 & 0.8722 & 0.8700 \\
\hline \multirow{2}{*}{ Tested Images } & \multicolumn{9}{|c|}{ FIIN Noise Denseness (\%) } \\
\hline & 50 & 55 & 60 & 65 & 70 & 75 & 80 & 85 & 90 \\
\hline Lena (TTSD) & 1.0000 & 1.0000 & 1.0000 & 1.0000 & 1.0000 & 1.0000 & 1.0000 & 1.0000 & 1.0000 \\
\hline Lena (QTSD) & 1.0000 & 1.0000 & 1.0000 & 1.0000 & 1.0000 & 1.0000 & 1.0000 & 1.0000 & 1.0000 \\
\hline M-cal(TTSD) & 1.0000 & 1.0000 & 1.0000 & 1.0000 & 1.0000 & 1.0000 & 1.0000 & 1.0000 & 1.0000 \\
\hline M-cal(QTSD) & 1.0000 & 1.0000 & 1.0000 & 1.0000 & 1.0000 & 1.0000 & 1.0000 & 1.0000 & 1.0000 \\
\hline Pepper (TTSD) & 1.0000 & 1.0000 & 1.0000 & 1.0000 & 1.0000 & 1.0000 & 1.0000 & 1.0000 & 1.0000 \\
\hline Pepper (QTSD) & 1.0000 & 1.0000 & 1.0000 & 1.0000 & 1.0000 & 1.0000 & 1.0000 & 1.0000 & 1.0000 \\
\hline Penta (TTSD) & 1.0000 & 1.0000 & 1.0000 & 1.0000 & 1.0000 & 1.0000 & 1.0000 & 1.0000 & 1.0000 \\
\hline Penta (QTSD) & 1.0000 & 1.0000 & 1.0000 & 1.0000 & 1.0000 & 1.0000 & 1.0000 & 1.0000 & 1.0000 \\
\hline Girl (TTSD) & 0.6551 & 0.6452 & 0.6345 & 0.6246 & 0.6122 & 0.6028 & 0.5905 & 0.5747 & 0.5577 \\
\hline Girl (QTSD) & 0.7132 & 0.7046 & 0.6961 & 0.6861 & 0.6747 & 0.6643 & 0.6505 & 0.6334 & 0.6137 \\
\hline Resolution (TTSD) & 0.1018 & 0.0907 & 0.0827 & 0.0722 & 0.0649 & 0.0506 & 0.0413 & 0.0294 & 0.0209 \\
\hline Resolution (QTSD) & 0.1025 & 0.0913 & 0.0833 & 0.0727 & 0.0650 & 0.0509 & 0.0415 & 0.0296 & 0.0209 \\
\hline Mandrill (TTSD) & 0.9992 & 0.9994 & 0.9994 & 0.9994 & 0.9994 & 0.9993 & 0.9991 & 0.9987 & 0.9984 \\
\hline Mandrill (QTSD) & 0.9996 & 0.9996 & 0.9996 & 0.9996 & 0.9997 & 0.9995 & 0.9993 & 0.9990 & 0.9987 \\
\hline House (TTSD) & 1.0000 & 1.0000 & 1.0000 & 1.0000 & 1.0000 & 1.0000 & 1.0000 & 1.0000 & 1.0000 \\
\hline House (QTSD) & 1.0000 & 1.0000 & 1.0000 & 1.0000 & 1.0000 & 1.0000 & 1.0000 & 1.0000 & 1.0000 \\
\hline Airplane (TTSD) & 1.0000 & 1.0000 & 1.0000 & 1.0000 & 1.0000 & 1.0000 & 1.0000 & 1.0000 & 1.0000 \\
\hline Airplane (QTSD) & 1.0000 & 1.0000 & 1.0000 & 1.0000 & 1.0000 & 1.0000 & 1.0000 & 1.0000 & 1.0000 \\
\hline Average(TTSD) & 0.8618 & 0.8595 & 0.8574 & 0.8551 & 0.8529 & 0.8503 & 0.8479 & 0.8448 & 0.8419 \\
\hline Average(QTSD) & 0.8684 & 0.8662 & 0.8643 & 0.8620 & 0.8599 & 0.8572 & 0.8546 & 0.8513 & 0.8481 \\
\hline
\end{tabular}

Table 3. The simulated result of overall noise and non-noise classification correction of the proposed QTSD filter

\begin{tabular}{|c|c|c|c|c|c|c|c|c|c|}
\hline \multirow{2}{*}{ Tested Images } & \multicolumn{9}{|c|}{ FIIN Noise Denseness (\%) } \\
\hline & 5 & 10 & 15 & 20 & 25 & 30 & 35 & 40 & 45 \\
\hline Lena (TTSD) & 1.0000 & 1.0000 & 1.0000 & 1.0000 & 1.0000 & 1.0000 & 1.0000 & 1.0000 & 1.0000 \\
\hline Lena (QTSD) & 1.0000 & 1.0000 & 1.0000 & 1.0000 & 1.0000 & 1.0000 & 1.0000 & 1.0000 & 1.0000 \\
\hline M-cal (TTSD) & 1.0000 & 1.0000 & 1.0000 & 1.0000 & 1.0000 & 1.0000 & 1.0000 & 1.0000 & 1.0000 \\
\hline M-cal (QTSD) & 1.0000 & 1.0000 & 1.0000 & 1.0000 & 1.0000 & 1.0000 & 1.0000 & 1.0000 & 1.0000 \\
\hline Pepper (TTSD) & 1.0000 & 1.0000 & 1.0000 & 1.0000 & 1.0000 & 1.0000 & 1.0000 & 1.0000 & 1.0000 \\
\hline Pepper (QTSD) & 1.0000 & 1.0000 & 1.0000 & 1.0000 & 1.0000 & 1.0000 & 1.0000 & 1.0000 & 1.0000 \\
\hline Penta (TTSD) & 1.0000 & 1.0000 & 1.0000 & 1.0000 & 1.0000 & 1.0000 & 1.0000 & 1.0000 & 1.0000 \\
\hline Penta (QTSD) & 1.0000 & 1.0000 & 1.0000 & 1.0000 & 1.0000 & 1.0000 & 1.0000 & 1.0000 & 1.0000 \\
\hline Girl (TTSD) & 0.8516 & 0.8527 & 0.8528 & 0.8516 & 0.8496 & 0.8467 & 0.8421 & 0.8374 & 0.8320 \\
\hline Girl (QTSD) & 0.8746 & 0.8733 & 0.8717 & 0.8699 & 0.8685 & 0.8668 & 0.8648 & 0.8626 & 0.8596 \\
\hline Resolution (TTSD) & 0.5973 & 0.5919 & 0.5865 & 0.5814 & 0.5764 & 0.5700 & 0.5678 & 0.5621 & 0.5556 \\
\hline Resolution (QTSD) & 0.5979 & 0.5923 & 0.5869 & 0.5820 & 0.5768 & 0.5705 & 0.5682 & 0.5623 & 0.5559 \\
\hline Mandrill (TTSD) & 0.9994 & 0.9994 & 0.9995 & 0.9996 & 0.9995 & 0.9996 & 0.9996 & 0.9996 & 0.9996 \\
\hline Mandrill (QTSD) & 0.9997 & 0.9997 & 0.9998 & 0.9998 & 0.9998 & 0.9998 & 0.9998 & 0.9998 & 0.9998 \\
\hline House (TTSD) & 1.0000 & 1.0000 & 1.0000 & 1.0000 & 1.0000 & 1.0000 & 1.0000 & 1.0000 & 1.0000 \\
\hline House (QTSD) & 1.0000 & 1.0000 & 1.0000 & 1.0000 & 1.0000 & 1.0000 & 1.0000 & 1.0000 & 1.0000 \\
\hline Airplane (TTSD) & 1.0000 & 1.0000 & 1.0000 & 1.0000 & 1.0000 & 1.0000 & 1.0000 & 1.0000 & 1.0000 \\
\hline Airplane (QTSD) & 1.0000 & 1.0000 & 1.0000 & 1.0000 & 1.0000 & 1.0000 & 1.0000 & 1.0000 & 1.0000 \\
\hline Average (TTSD) & 0.9387 & 0.9382 & 0.9376 & 0.9370 & 0.9362 & 0.9351 & 0.9344 & 0.9332 & 0.9319 \\
\hline Average (QTSD) & 0.9413 & 0.9406 & 0.9398 & 0.9391 & 0.9383 & 0.9374 & 0.9370 & 0.9361 & 0.9350 \\
\hline \multirow{2}{*}{ Tested Images } & \multicolumn{9}{|c|}{ FIIN Noise Denseness (\%) } \\
\hline & 5 & 10 & 15 & 20 & 25 & 30 & 35 & 40 & 45 \\
\hline Lena (TTSD) & 1.0000 & 1.0000 & 1.0000 & 1.0000 & 1.0000 & 1.0000 & 1.0000 & 1.0000 & 1.0000 \\
\hline Lena (QTSD) & 1.0000 & 1.0000 & 1.0000 & 1.0000 & 1.0000 & 1.0000 & 1.0000 & 1.0000 & 1.0000 \\
\hline M-cal (TTSD) & 1.0000 & 1.0000 & 1.0000 & 1.0000 & 1.0000 & 1.0000 & 1.0000 & 1.0000 & 1.0000 \\
\hline M-cal (QTSD) & 1.0000 & 1.0000 & 1.0000 & 1.0000 & 1.0000 & 1.0000 & 1.0000 & 1.0000 & 1.0000 \\
\hline Pepper (TTSD) & 1.0000 & 1.0000 & 1.0000 & 1.0000 & 1.0000 & 1.0000 & 1.0000 & 1.0000 & 1.0000 \\
\hline Pepper (QTSD) & 1.0000 & 1.0000 & 1.0000 & 1.0000 & 1.0000 & 1.0000 & 1.0000 & 1.0000 & 1.0000 \\
\hline Penta (TTSD) & 1.0000 & 1.0000 & 1.0000 & 1.0000 & 1.0000 & 1.0000 & 1.0000 & 1.0000 & 1.0000 \\
\hline Penta (QTSD) & 1.0000 & 1.0000 & 1.0000 & 1.0000 & 1.0000 & 1.0000 & 1.0000 & 1.0000 & 1.0000 \\
\hline Girl (TTSD) & 0.8276 & 0.8226 & 0.8173 & 0.8123 & 0.8061 & 0.8014 & 0.7953 & 0.7874 & 0.7789 \\
\hline Girl (QTSD) & 0.8566 & 0.8523 & 0.8481 & 0.8431 & 0.8374 & 0.8322 & 0.8253 & 0.8167 & 0.8069 \\
\hline Resolution (TTSD) & 0.5509 & 0.5454 & 0.5414 & 0.5361 & 0.5325 & 0.5253 & 0.5207 & 0.5147 & 0.5105 \\
\hline
\end{tabular}


Table 3. The simulated result of overall noise and non-noise classification correction of the proposed QTSD filter (continue)

\begin{tabular}{|c|c|c|c|c|c|c|c|c|c|}
\hline \multirow{2}{*}{ Tested Images } & \multicolumn{9}{|c|}{ FIIN Noise Denseness (\%) } \\
\hline & 5 & 10 & 15 & 20 & 25 & 30 & 35 & 40 & 45 \\
\hline Resolution (QTSD) & 0.5513 & 0.5457 & 0.5417 & 0.5364 & 0.5325 & 0.5255 & 0.5208 & 0.5148 & 0.5105 \\
\hline Mandrill (TTSD) & 0.9996 & 0.9997 & 0.9997 & 0.9997 & 0.9997 & 0.9997 & 0.9996 & 0.9994 & 0.9992 \\
\hline Mandrill (QTSD) & 0.9998 & 0.9998 & 0.9998 & 0.9998 & 0.9999 & 0.9998 & 0.9997 & 0.9995 & 0.9994 \\
\hline House (TTSD) & 1.0000 & 1.0000 & 1.0000 & 1.0000 & 1.0000 & 1.0000 & 1.0000 & 1.0000 & 1.0000 \\
\hline House (QTSD) & 1.0000 & 1.0000 & 1.0000 & 1.0000 & 1.0000 & 1.0000 & 1.0000 & 1.0000 & 1.0000 \\
\hline Airplane (TTSD) & 1.0000 & 1.0000 & 1.0000 & 1.0000 & 1.0000 & 1.0000 & 1.0000 & 1.0000 & 1.0000 \\
\hline Airplane (QTSD) & 1.0000 & 1.0000 & 1.0000 & 1.0000 & 1.0000 & 1.0000 & 1.0000 & 1.0000 & 1.0000 \\
\hline Average (TTSD) & 0.9309 & 0.9297 & 0.9287 & 0.9276 & 0.9265 & 0.9252 & 0.9239 & 0.9224 & 0.9209 \\
\hline Average (QTSD) & 0.9342 & 0.9331 & 0.9322 & 0.9310 & 0.9300 & 0.9286 & 0.9273 & 0.9257 & 0.9241 \\
\hline
\end{tabular}

Table 4. The simulated result of proposed noise suppressing algorithms (PSNR in $\mathrm{dB}$ )

\begin{tabular}{|c|c|c|c|c|c|c|c|c|c|}
\hline \multirow{2}{*}{ Tested Images } & \multicolumn{9}{|c|}{ FIIN Noise Denseness (\%) } \\
\hline & 5 & 10 & 15 & 20 & 25 & 30 & 35 & 40 & 45 \\
\hline Lena (TTSD) & 44.1437 & 40.5683 & 38.4735 & 37.0012 & 35.4691 & 34.6549 & 33.6396 & 32.7912 & 32.1537 \\
\hline Lena (QTSD) & 44.1437 & 40.5683 & 38.4735 & 37.0012 & 35.4691 & 34.6549 & 33.6396 & 32.7912 & 32.1537 \\
\hline M-cal (TTSD) & 33.8265 & 30.7515 & 28.7241 & 27.3321 & 26.0929 & 25.1559 & 24.3449 & 23.4485 & 22.6226 \\
\hline M-cal (QTSD) & 33.8265 & 30.7515 & 28.7241 & 27.3321 & 26.0929 & 25.1559 & 24.3449 & 23.4485 & 22.6226 \\
\hline Pepper (TTSD) & 44.6517 & 40.9933 & 39.0767 & 37.2222 & 35.6168 & 34.6218 & 33.3610 & 32.7192 & 31.5482 \\
\hline Pepper (QTSD) & 44.6517 & 40.9933 & 39.0767 & 37.2222 & 35.6168 & 34.6218 & 33.3610 & 32.7192 & 31.5482 \\
\hline Penta (TTSD) & 41.5360 & 38.4091 & 36.4657 & 34.8211 & 33.6704 & 32.8752 & 31.9126 & 31.0808 & 30.3577 \\
\hline Penta (QTSD) & 41.5360 & 38.4091 & 36.4657 & 34.8211 & 33.6704 & 32.8752 & 31.9126 & 31.0808 & 30.3577 \\
\hline Girl (TTSD) & 40.9275 & 39.0460 & 37.1363 & 36.0495 & 35.1385 & 34.4868 & 33.2885 & 33.1268 & 32.4662 \\
\hline Girl (QTSD) & 42.2002 & 39.9614 & 37.9219 & 37.0343 & 35.4994 & 35.1516 & 33.9162 & 33.5191 & 32.9332 \\
\hline Resolution (TTSD) & 10.8895 & 10.7693 & 10.7106 & 10.6314 & 10.5477 & 10.3913 & 10.4712 & 10.0244 & 10.1557 \\
\hline Resolution (QTSD) & 10.9906 & 10.8685 & 10.7845 & 10.7406 & 10.6264 & 10.4790 & 10.5206 & 10.0927 & 10.2053 \\
\hline Mandrill (TTSD) & 35.6049 & 32.8156 & 31.1219 & 29.8013 & 28.6195 & 27.6662 & 26.9878 & 26.2291 & 25.5535 \\
\hline Mandrill (QTSD) & 35.6079 & 32.8174 & 31.1234 & 29.8029 & 28.6203 & 27.6656 & 26.9890 & 26.2294 & 25.5545 \\
\hline House (TTSD) & 43.4424 & 39.2905 & 36.8436 & 35.4475 & 33.8891 & 33.1098 & 31.8028 & 31.4430 & 30.6868 \\
\hline House (QTSD) & 43.4424 & 39.2905 & 36.8436 & 35.4475 & 33.8891 & 33.1098 & 31.8028 & 31.4430 & 30.6868 \\
\hline Airplane (TTSD) & 44.4395 & 40.0741 & 38.0206 & 36.4108 & 35.1259 & 33.9876 & 33.1300 & 32.1944 & 31.6389 \\
\hline Airplane (QTSD) & 44.4395 & 40.0741 & 38.0206 & 36.4108 & 35.1259 & 33.9876 & 33.1300 & 32.1944 & 31.6389 \\
\hline \multirow{2}{*}{ Tested Images } & \multicolumn{9}{|c|}{ FIIN Noise Denseness (\%) } \\
\hline & 50 & 55 & 60 & 65 & 70 & 75 & 80 & 85 & 90 \\
\hline Lena (TTSD) & 31.4062 & 30.7008 & 29.9415 & 29.3282 & 28.6491 & 27.7192 & 27.0346 & 26.4298 & 24.9663 \\
\hline Lena (QTSD) & 31.4062 & 30.7008 & 29.9415 & 29.3282 & 28.6491 & 27.7192 & 27.0346 & 26.4298 & 24.9663 \\
\hline M-cal (TTSD) & 22.0194 & 21.4728 & 20.8748 & 20.3634 & 19.6682 & 19.0352 & 18.4647 & 17.8237 & 16.9359 \\
\hline M-cal (QTSD) & 22.0194 & 21.4728 & 20.8748 & 20.3634 & 19.6682 & 19.0352 & 18.4647 & 17.8237 & 16.9359 \\
\hline Pepper (TTSD) & 31.2424 & 30.3661 & 29.5347 & 28.9315 & 28.2225 & 27.3633 & 26.4359 & 25.3887 & 24.1841 \\
\hline Pepper (QTSD) & 31.2424 & 30.3661 & 29.5347 & 28.9315 & 28.2225 & 27.3633 & 26.4359 & 25.3887 & 24.1841 \\
\hline Penta (TTSD) & 29.7195 & 29.0494 & 28.4674 & 27.7912 & 27.1497 & 26.5101 & 25.7817 & 25.0132 & 23.9414 \\
\hline Penta (QTSD) & 29.7195 & 29.0494 & 28.4674 & 27.7912 & 27.1497 & 26.5101 & 25.7817 & 25.0132 & 23.9414 \\
\hline Girl (TTSD) & 31.1927 & 30.7870 & 30.4537 & 29.5127 & 29.1430 & 28.0601 & 27.3623 & 26.1170 & 24.5832 \\
\hline Girl (QTSD) & 31.8293 & 31.3880 & 31.0127 & 30.2264 & 29.5584 & 29.0116 & 28.3650 & 27.1897 & 26.0562 \\
\hline Resolution (TTSD) & 9.9008 & 9.8114 & 9.4654 & 9.6342 & 9.6163 & 9.3103 & 9.3463 & 8.9960 & 8.3406 \\
\hline Resolution (QTSD) & 9.9958 & 9.8880 & 9.6064 & 9.6928 & 9.6248 & 9.3385 & 9.3981 & 9.0743 & 8.3406 \\
\hline Mandrill (TTSD) & 24.9107 & 24.3501 & 23.8627 & 23.3415 & 22.8220 & 22.2262 & 21.7492 & 21.0479 & 20.2954 \\
\hline Mandrill (QTSD) & 24.9109 & 24.3500 & 23.8625 & 23.3416 & 22.8219 & 22.2264 & 21.7491 & 21.0466 & 20.2954 \\
\hline House (TTSD) & 29.7906 & 29.4475 & 28.6620 & 27.2870 & 27.0843 & 25.9919 & 25.5637 & 23.8973 & 23.3652 \\
\hline House (QTSD) & 29.7906 & 29.4475 & 28.6620 & 27.2870 & 27.0843 & 25.9919 & 25.5637 & 23.8973 & 23.3652 \\
\hline Airplane (TTSD) & 30.9843 & 30.0582 & 29.1667 & 28.8364 & 28.2869 & 27.4683 & 26.7261 & 25.7139 & 24.2702 \\
\hline Airplane (QTSD) & 30.9843 & 30.0582 & 29.1667 & 28.8364 & 28.2869 & 27.4683 & 26.7261 & 25.7139 & 24.2702 \\
\hline
\end{tabular}

From these simulation results, classification correctness of the proposed QTSD filter has equally efficacy with the classical TTSD for six noise photographs (Lena, M-calendar, Pepper, Pentagon, House, Airplane), which is the non-full dynamic range elements $\left(0<x_{i, j}<255\right)$ however classification correctness of the proposed QTSD has ultimately efficacy with the classical TTSD for other three noise photographs (Girl, Resolution, Mandrill), which is the full dynamic range elements $\left(0<x_{i, j}<255\right)$. Next, the $2^{\text {nd }}$ experimental simulation of efficacy (in PSNR) of noise suppressing algorithm found on QTSD filter [28], which is correlatively analyzed with the classical TTSD filter [28], are illustrated in Table 4 under noise denseness from $5 \%$ to $90 \%$. From these simulation results, the efficacy of noise suppressing algorithm found on QTSD filter and modified AMF [26]-[28] has equally efficacy with the classical TTSD for six noise photographs (lena, m-calendar, pepper, pentagon, house, airplane). However, classification correctness of the proposed QTSD has ultimately efficacy with the classical TTSD for other three noise photographs. (Girl, 
Resolution, Mandrill). Because of page obstruction, a few part of simulation results of Girl that are suppressed by QTSD, TTSD and other classical suppressing methods can be illustrated as Figure 2.

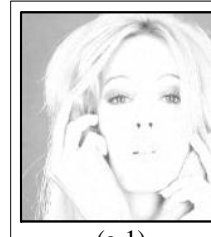

(a-1)

Non-Noise Photo

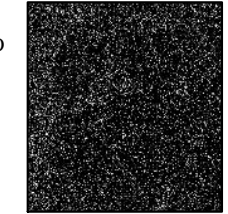

(a-2) $(\mathrm{D}=20 \%)$

Outlier Photo
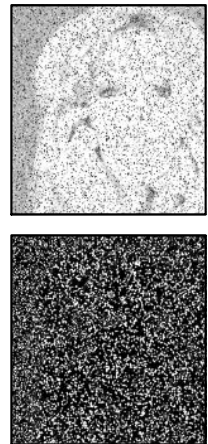

(a-3)

Gauss-Filter
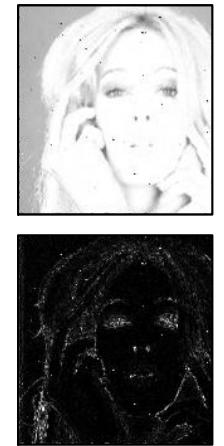

(a-4)

SMF
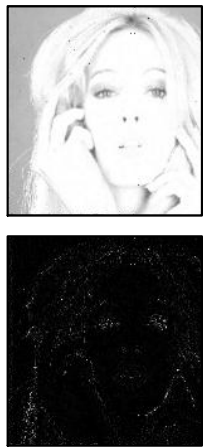

(a-5)

AMF
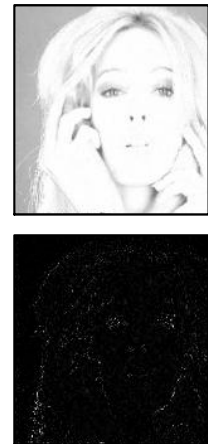

(a-6)

TTSD Filter
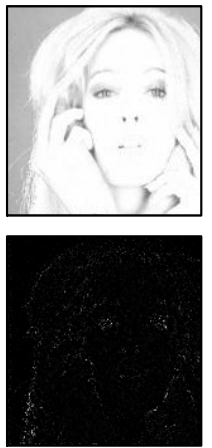

(a-7)

QTSD Filter

$(\mathrm{PSNR}=10.6567 \mathrm{~dB})(\mathrm{PSNR}=13.9747 \mathrm{~dB})(\mathrm{PSNR}=25.5153 \mathrm{~dB})(\mathrm{PSNR}=32.0437 \mathrm{~dB})(\mathrm{PSNR}=36.0495 \mathrm{~dB})(\mathrm{PSNR}=37.0343 \mathrm{~dB})$

Figure 2. The few visualization simulation results of proposed noise suppressing algorithm and earlier noise suppressing algorithms

\section{CONCLUSION}

For suppressing FIIN, this article aims to propose the novel noise classification technique found on QTSD filter, which is ultimately improved from the outstanding TTSD filter. From these simulation results of noise classification point of view, the proposed noise classification techniques found on QTSD can definitely produce the better efficacy than the earlier noise classification techniques. Furthermore, from these simulation results of noise suppressing point of view, the proposed noise suppressing algorithm found on QTSD and modified AMF can definitely produce the better efficacy than the earlier noise suppressing algorithm. For afterward research, we will investigate and invent the better efficacy noise suppressing algorithm found on QTSD and better efficacy noise recovering technique.

\section{APPENDIX}

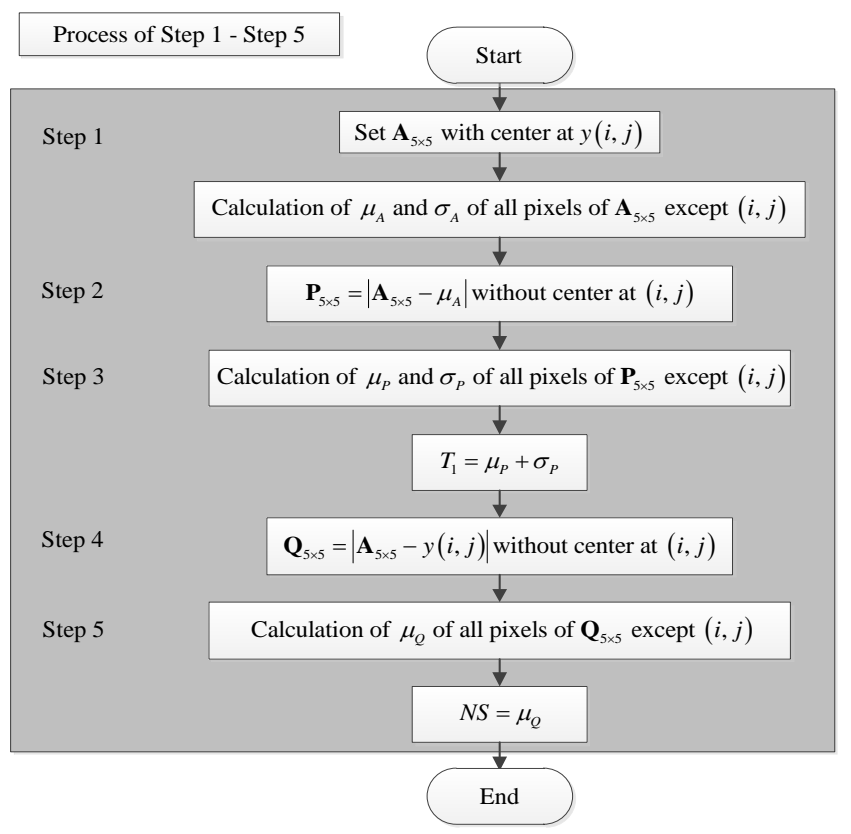

Figure 1. The extensive sub-process computation of the proposed QTSD filter 


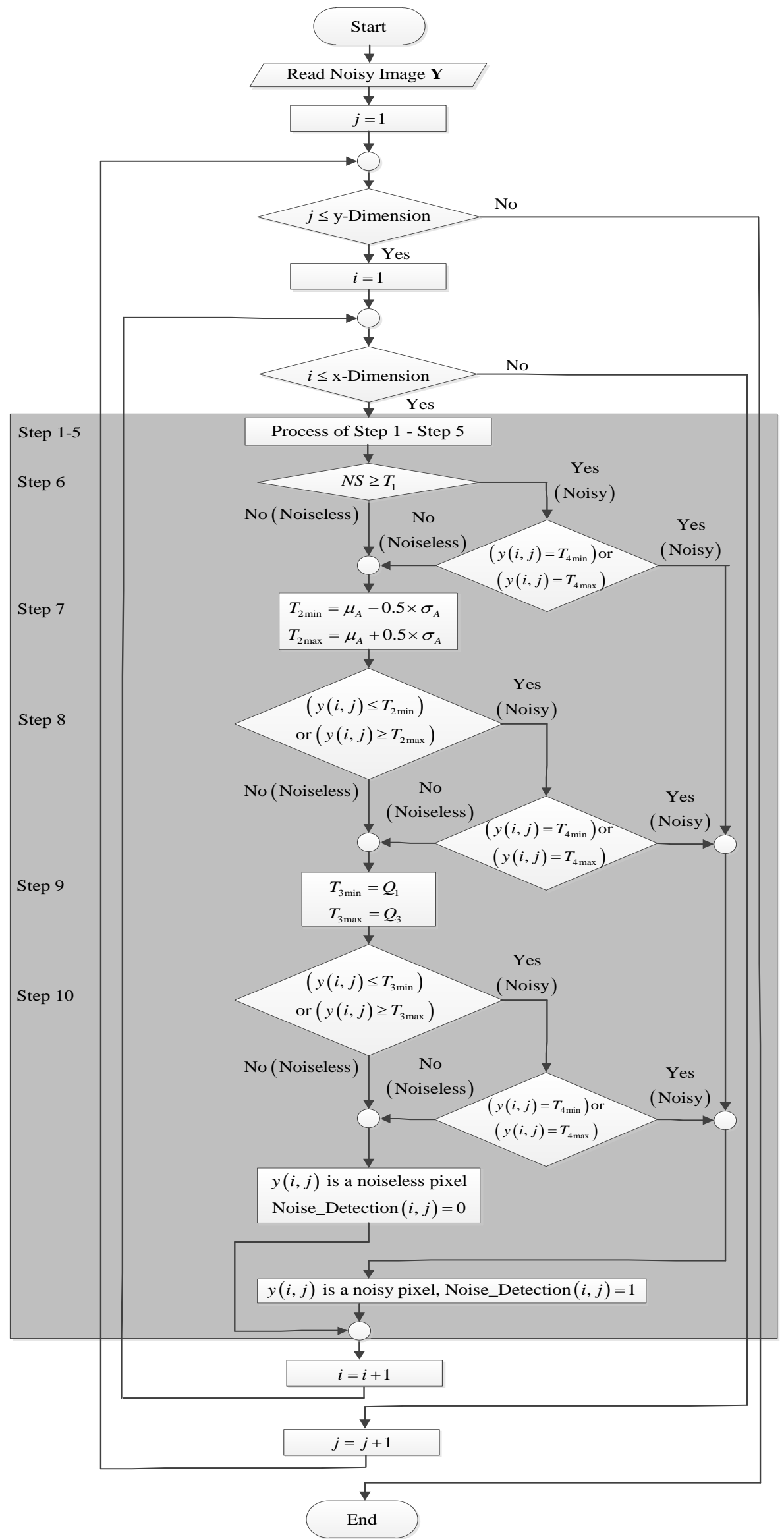

Figure 1. The extensive sub-process computation of the proposed QTSD filter (continue) 


\section{ACKNOWLEDGEMENTS}

The portions of this research work were presented at the 43th Electrical Engineering Conference (EECON-43), EEAAT ((Electrical Engineering Academic Association (Thailand), Thailand, Oct. 2020, as "The Novel Outlier Detection Algorithm Based on QTSD (Quadruple Threshold Statistical Detection) Filter for FIIN (Fix Intensity Impulse Noise)".

\section{REFERENCES}

[1] R. C. Gonzalez and R. E. Woods, Digital Image Processing, Prentice-Hall, Upper Saddle River, NJ, USA, 2nd edition, 2002.

[2] A. S. M. Shafi, and Mohammad Motiur Rahman, "Decomposition of color wavelet with higher order statistical texture and convolutional neural network features set based classification of colorectal polyps from video endoscopy," International Journal of Electrical and Computer Engineering (IJECE), vol. 10, no. 3, pp. 2986-2996, June 2020, doi: 10.11591/ijece.v10i3.pp2986-2996.

[3] Susama Bagchi, Kim Gaik Tay, Audrey Huong, andSanjoy Kumar Debnath, "Image processing and machine learning techniques used in computer-aided detection system for mammogram screening-A review," International Journal of Electrical and Computer Engineering (IJECE), vol. 10, no. 3, pp. 2336-2348, June 2020, doi: 10.11591/ijece.v10i3.pp2336-2348.

[4] Nur Dalila Abdullah, Ummi Raba'ah Hashim, Sabrina Ahmad, and Lizawati Salahuddin, "Analysis of texture features for wood defect classification," Bulletin of Electrical Engineering and Informatics (BEEI), vol. 9, no. 1, pp. 121-128, 2020, doi: doi.org/10.11591/eei.v9i1.1553.

[5] Alaa Jabbar Qasim, Roshidi Din, and Farah Qasim Ahmed Alyousuf, "Review on techniques and file formats of image compression," Bulletin of Electrical Engineering and Informatics (BEEI), vol. 9, no. 2, pp. 602-610, April 2020, doi: 10.11591/eei.v9i2.2085.

[6] Patil V H, Kharate G K, and Kamlapur S M, "Super resolution imaging needs better registration for better quality results," Bulletin of Electrical Engineering and Informatics (BEEI), vol. 1, no. 1, pp. 43-50, March 2012.

[7] Chengzhi Deng, Juanjuan Liu, Wei Tian, Shengqian Wang, Huasheng Zhu, and Shaoquan Zhang, "Image superresolution reconstruction based on L1/2 sparsity," Bulletin of Electrical Engineering and Informatics (BEEI), vol. 3, no. 3, pp. September 2014.

[8] Darun Kesrarat, Kornkamol Thakulsukanant, and Vorapoj Patanavijit, "A novel elementary spatial expanding scheme form on SISR method with modifying Geman\&McClure function," TELKOMNIKA, vol. 17, no. 5, pp. 2554-2560, Oct 2019, doi: 10.12928/TELKOMNIKA.v17i5.12799.

[9] Srinivasa Perumal Ramalingam, Nadesh R. K., and SenthilKumar N. C., "Robust face recognition using enhanced local binary pattern," Bulletin of Electrical Engineering and Informatics (BEEI), vol. 7, no. 1, pp. 96-101, March 2018, doi: 10.11591/eei.v7i1.761.

[10] J. Astola, P. Haavisto, and Y. Neuvo, "Vector median filters," in Proceedings of the IEEE, 1990, vol. 78, no. 4, pp 678-689, doi: 10.1109/5.54807.

[11] Om Prakash Verma and Nitin Sharma, "Intensity preserving cast removal in color images using particle swarm optimization," International Journal of Electrical and Computer Engineering (IJECE), vol. 7, no. 5, pp. 25812595, October 2017, doi: 10.11591/ijece.v7i5.pp2581-2595.

[12] Madina Hamiane and Fatema Saeed, "SVM classification of MRI brain images for computer-assisted diagnosis," International Journal of Electrical and Computer Engineering (IJECE), vol. 7, no. 5, pp. 2555-2564, October 2017, doi: 10.11591/ijece.v7i5.pp2555-2564.

[13] Asem Khmag, Sami Ghoul, Syed Abdul Rahman Al-Haddad, and Noraziahtulhidayu Kamarudin, "Noise level estimation for digital images using local statistics and its applications to noise removal," TELKOMNIKA, vol. 16, no. 2, pp. 915-924, April 2018, doi: 10.12928/telkomnika.v16i2.9060.

[14] K. Arun Sai, and K. Ravi, "An efficient filtering technique for denoising colour images," International Journal of Electrical and Computer Engineering (IJECE), vol. 8, no. 5, pp. 3604-3608, October 2018, doi: 10.11591/ijece.v8i5.pp3604-3608.

[15] Zayed M. Ramadan, "Effect of kernel size on Wiener and Gaussian image filtering," TELKOMNIKA, vol. 17, no. 3, pp. 1455-1460, June 2019, doi: 10.12928/telkomnika.v17i3.11192.

[16] Jufriadif Na'am, Johan Harlan, Rosda Syelly, and Agung Ramadhanu, "Filter technique of medical image on multiple morphological gradient (MMG) method," TELKOMNIKA, vol. 17, no. 3, pp. June 2019, doi: 10.12928/telkomnika.v17i3.9722.

[17] Bilal Charmouti, et al., "An overview of the fundamental approaches that yield several image denoising techniques," TELKOMNIKA, vol. 17, no. 6, pp. 2959-2967, December 2019, doi: 10.12928/telkomnika.v17i6.11301.

[18] Lanani Abderrahim, Meghriche Salama, and Djouambi Abdelbaki, "Novel design of a fractional wavelet and its application to image denoising," Bulletin of Electrical Engineering and Informatics (BEEI), vol. 9, no. 1, pp. 129140, February 2020, doi: 10.11591/eei.v9i1.1548.

[19] Yasin Yousif Al-Aboosi, Radhi Sehen Issa, and Ali Khalid Jassim, "Image denosing in underwater acoustic noise using discrete wavelet transform with different noise level estimation," TELKOMNIKA, vol. 18, no. 3, pp. 14391446, June 2020, doi: 10.12928/telkomnika.v18i3.14381. 
[20] Pitas I., Venetsanopoulos A.N. "Median Filters," in Nonlinear Digital Filters, The Springer International Series in Engineering and Computer Science (VLSI, Computer Architecture and Digital Signal Processing), vol 84. Springer, Boston, MA, 1990, doi: 10.1007/978-1-4757-6017-0_4.

[21] H. Hwang and R. A. Haddad, "Adaptive median filters new algorithms and results," IEEE Transactions on Image Processing, vol. 4, no. 4, pp. 499-502, 1995, doi: 10.1109/83.370679.

[22] S. Rajkumar, and G. Malathi, "An efficient image denoising approach for the recovery of impulse noise," Bulletin of Electrical Engineering and Informatics (BEEI), vol. 6, no. 3, pp. 281-286, September 2017, doi: 10.11591/eei.v6i3.680.

[23] V. Patanavijit, "Denoising performance analysis of adaptive decision based inverse distance weighted interpolation (DBIDWI) algorithm for salt and pepper noise," Indonesian Journal of Electrical Engineering and Computer Science, vol. 15, no. 2, pp. 804-813, 2019, doi: 10.11591/ijeecs.v15.i2.pp804-813.

[24] Vasanth Kishorebabu, Ganesan Packyanathan, Harikrishna Kamatham, and Vishnu Shankar, "An adaptive decision based interpolation scheme for the removal of high density salt and pepper noise in images," EURASIP Journal on Image and Video Processing, vol. 2017, 2017, doi: 10.1186/s13640-017-0215-0.

[25] Vorapoj Patanavijit, and Kornkamol Thakulsukanant, "The statistical analysis of random-valued impulse noise detection techniques based on the local image characteristic: ROAD, ROLD and RORD," Indonesian Journal of Electrical Engineering and Computer Science, vol. 15, no. 2, pp. 794-803, 2019, doi: 10.11591/ijeecs.v15.i2.pp794-803.

[26] Neeti Singh and Umamaheswari Oorkavalan, "Triple threshold statistical detection filter for removing high density random-valued impulse noise in images," EURASIP Journal on Image and Video Processing, vol. 2018, no 1, p. 22, 2018, doi: 10.1186/s13640-018-0263-0.

[27] K. Thakulsukanant and V. Patanavijit, "Correlation analysis inspection of noise obliteration operation stand on TTSD filter under random-valued impulse noise circumstances," EECON-36, EEAAT, Greenery Resort, Thailand, Oct. 2019.

[28] V. Patanavijit and K. Thakulsukanant, "The Novel Outlier Detection Algorithm Based on QTSD (Quadruple Threshold Statistical Detection) Filter for FIIN (Fix Intensity Impulse Noise)," EECON-43, EEAAT, Phitsanulok, Thailand, Oct. 2020. 